\title{
Juguetes, tecnología y discapacidad. Ingeniería aplicada al diseño de juguetes adaptados
}

Sergio Daniel Díaz*, Said Pinzón Castañeda**

\section{Resumen}

El presente artículo expone el interés del semillero de investigación Electrocun, con apoyo del grupo de investigación IDECUN, de la Corporación Unificada de Educación Superior, CUN, de incursionar en el diseño y desarrollo de juguetes adaptados para niños en condición de discapacidad. Este artículo tiene como objetivo presentar la primera etapa del diseño de un prototipo de juego para niños con discapacidad cognitiva y trastorno de déficit de atención e hiperactividad (TDAH). El alcance del presente documento abarca un contexto acerca de qué es la discapacidad cognitiva y el TDAH, de cómo la ingeniería apoya el diseño y desarrollo de juguetes adaptados con fines lúdicos y terapéuticos y una descripción de la fase inicial de desarrollo del prototipo objetivo de este proyecto.

Palabras Clave: adaptaciones tecnológicas, discapacidad cognitiva, diseño universal, ingeniería biomédica, juguete adaptado

\begin{abstract}
This article exposes the interest of the Electrocun group with the support of the IDECUN research group of Corporación Unificada de Educación Superior, CUN, to venture into the design and development of toys adapted for children in disability condition. This descriptive article presents the first stage of design of a functional prototype of a game adapted for children with cognitive disabilities and with attention deficit hyperactivity disorder (ADHD). The scope of this document covers a context of what is cognitive disability and ADHD, how engineering supports the design and development of toys adapted for recreational and therapeutic purposes, and a description of the initial development phase of the prototype objective of this project.
\end{abstract}

Keywords: Adapted Toy. Cognitive disability, Biomedical Engineering, Technological Adaptations, Universal Design

* Corporación Unificada Nacional de Educación Superior - CUN. Contacto: sergio_diaz@cun.edu.co
** Corporación Unificada Nacional de Educación Superior - CUN. Contacto: said_pinzon@cun.edu.co 


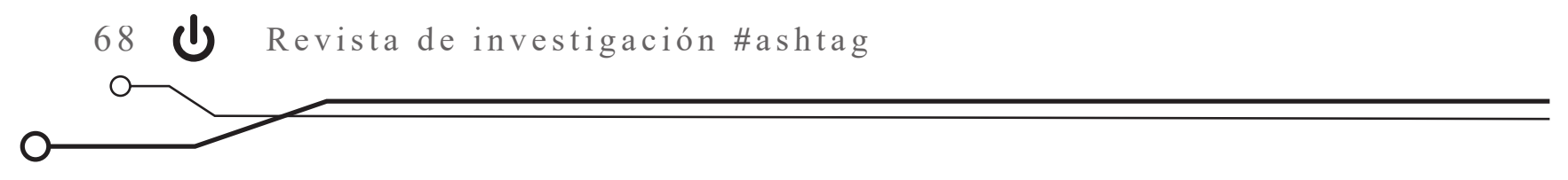

\section{Introducción}

La Organización Mundial de la Salud, OMs, estableció un marco de referencia sobre la salud y la discapacidad, en la Clasificación Internacional del Funcionamiento, comúnmente conocida como CIF. En la CIF el concepto de discapacidad no es la simple caracterización de algunas personas, sino que es un fenómeno muy complejo que atañe, sistémicamente, a todo ser humano, en cuanto a la interacción entre su propia condición de salud y su contexto personal y ambiental. Así, el término discapacidad pretende ser una generalidad que abarca: 1) las deficiencias propias en la estructura o el funcionamiento corporal de un individuo, 2) sus limitaciones en la realización de acciones y labores, y 3) las restricciones de su participación en situaciones vitales según el estándar social de su respectiva cultura. (Organización Mundial de la Salud, 2001).

En el informe mundial sobre la discapacidad, la OMs afirma que más de mil millones de personas, es decir, un $15 \%$ de la población mundial, padece alguna forma de discapacidad; entre 110 millones y 190 millones de adultos tienen dificultades considerables para funcionar. La discapacidad es cada día más preocupante, pues su prevalencia está en aumento, debido a que la población de adultos mayores también está incrementando, y sus condiciones de salud los hace más propensos a la discapacidad. Adicionalmente, el ritmo de la vida contemporánea ha incrementado el desarrollo de enfermedades crónicas como la diabetes, las enfermedades cardiovasculares, el cáncer y trastornos en la salud mental (Organización Mundial de la Salud, 2011).

En todo el mundo, las personas con discapacidad tienen peores resultados académicos, una menor participación económica y unas tasas de pobreza más altas que las personas sin discapacidad. En parte, ello es consecuencia de los obstáculos que entorpecen el acceso de las personas con discapacidad a servicios de salud, a la educación, el empleo, el transporte, o la información. Esas dificultades se exacerban en las comunidades menos favorecidas.

Para el caso de la población infantil, la situación no es muy diferente, ya que apenas ahora se está hablando de un enfoque inclusivo en las escuelas y en espacios que antes eran relegados para niños con un tipo de condición especial. El término inclusión también abarca los objetos y ayudas técnicas que le permiten a esta población tener una comunicación con mayor independencia y calidad de vida (Moreno y Martínez 2014).

De acuerdo con el portal Discapacidad Colombia, a nivel mundial se estima que 93 millones de niños, o 1 de cada 20 entre los que tienen hasta 14 años, viven con una discapacidad moderada o severa. En la mayoría de los países con ingresos bajos y medios, los niños con discapacidades tienen más probabilidades de no escolarizarse que cualquier otro grupo de niños. Las tasas de primera inscripción escolar de niños con discapacidades son muy bajas. Incluso cuando asisten a la escuela, los niños que sufren discapacidades son más susceptibles a abandonar su escolarización o finalizarla prematuramente (Discapacidad Colombia, 2017).

De acuerdo con expertos en juego y juguetes, del Centro Tecnológico AIJu, "el juego permite desarrollar la propia capacidad física y mental. Es una fuente de autoafirmación, satisfacción y placer. Jugar significa ser activo y prepararse para la vida adulta. Sin duda una carencia importante 
de juego en la infancia ocasionará un desarrollo incorrecto e incompleto de la personalidad del niño" (Costa et al., 2007, pág. 3).

En ese orden de ideas, dentro de las ayudas técnicas o tecnologías adaptadas, se resalta un término aplicado a estos desarrollos: diseño universal o diseño para todos. El diseño universal consiste en la percepción y creación de diversos productos, entornos y servicios, para que puedan ser utilizados por el mayor número posible de personas, sin necesidad de adaptaciones o de proyectos especializados. El diseño universal beneficia a personas de todas las edades y capacidades. No hace separación entre las personas, sino que busca la adecuación para todos en todo

\section{El prototipo y su metodología}

\section{Planteamiento del problema}

Hoy en día, la tecnología avanza a pasos agigantados. Esto permite a cualquier persona tener un amplio acceso a diversas temáticas, que antes se creían exclusivos para expertos altamente capacitados en diferentes campos. Con la aparición del internet y demás herramientas para compartir información de todo tipo a nivel global (correo, chats, redes sociales, etc.), ha crecido el desarrollo de dispositivos lúdicos que tienen enfoques educativos, sociales, empresariales y familiares (De Miguel, 2006).

Existen miles de sitios web, aplicaciones y dispositivos didácticos electrónicos que se enfocan en la ayuda a personas que tienen enfermedades asociadas a la discapacidad cognitiva. Diversos estudios han dejado ver que mediante la estimulación con juegos didácticos las personas momento con el mismo diseño, o bien ofreciendo opciones para diferentes necesidades. Con esta aplicación se ha buscado diseñar tecnología que pueda ser accesible para cualquier persona sin que tenga que tener una condición especial.

Uno de los objetivos de la ingeniería es el diseño y desarrollo de tecnologías que hagan más fáciles y amigables las actividades diarias, entre ellas las actividades lúdicas, además de ofrecer una nueva alternativa para el bienestar de las personas con limitaciones, a través de los juegos, de modo que estos se conviertan en medios y herramientas terapéuticas de acuerdo con cada discapacidad y diseño (Arévalo, 2007).

retienen un 25 más de información con respecto a la forma tradicional de aprendizaje. Además, los pacientes expuestos a esta clase de juegos presentan mejoras notorias en sus capacidades motrices y mentales (Discpacnet, s. f.).

Por lo anterior, surge la idea de iniciar investigaciones y desarrollos de dispositivos lúdicos para personas con discapacidad. En este primer acercamiento, se propuso el desarrollo de un prototipo funcional de juego didáctico, cuya finalidad es hacer una interacción lúdica con niños en edad escolar que tengan algún tipo de discapacidad cognitiva, motora o déficit de atención, que posibilite la estimulación físico-motora dentro de un cuadro terapéutico y de seguimiento, para establecer el impacto ocasionado por el uso de este tipo de juegos. 


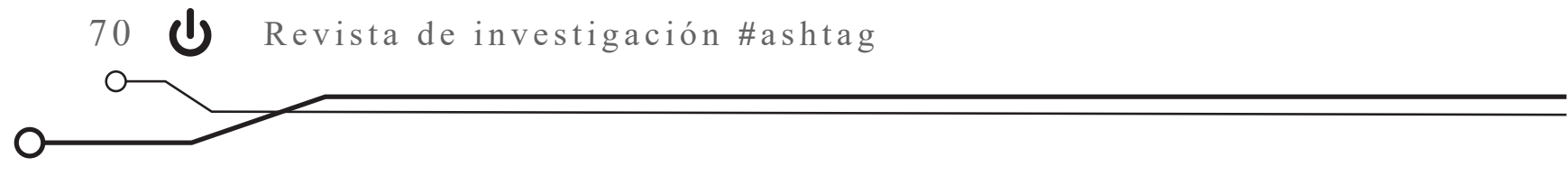

\section{Metodología}

La metodología que se propone en el proyecto corresponde a un ciclo de elaboración fundamental con 3 fases que darán forma al prototipo y mostrará sus implicaciones en la problemática propuesta. Estas incluyen las características de uso clínico, si el dispositivo será solo lúdico o si también tendrá un componente terapéutico, que sea medible de acuerdo con protocolos de rehabilitación definidos.

\section{Primera fase: diagnóstico para identificación de variables}

En esta fase se realizará la identificación de las variables o factores involucrados en el desarrollo del prototipo propuesto junto a las variables clínicas. También se procederá a definir el caso de

\section{Segunda fase: diseño del prototipo}

En esta fase se construirá el documento maestro de diseño con las variables y características de funcionalidad y uso definidas, Para este caso, las variables clínicas aún están por definir. También se describe el desarrollo que se realizó para la construcción del diseño del dispositivo electrónico, en el que se tendrá en cuenta las variables asociadas en la primera fase. Se define los esquemas correspondientes al hardware (diagrama de bloques) y software (diagrama de flujo).

También se considerarán los principios de diseño universal, además de las variables terapéuticas que se definan con los profesionales en rehabilitación, para que así las siguientes fases tengan un desarrollo de acuerdo con la finalidad y uso del dispositivo (Sevilla, 2011):

1. Uso equitativo, es decir, que se proporcione iguales maneras de uso y sea atractivo para todos los usuarios.

2. Flexibilidad de uso, es decir, que se adapte a las habilidades propias del usuario. estudio como un proyecto de investigación, cuyo objetivo fundamental es iniciar con el desarrollo de dispositivos tecnológicos y ayudas técnicas para personas en situación de discapacidad.

3. Uso simple e intuitivo, es decir, que sea fácil de entender según el nivel de alfabetización del usuario, sin complejidades innecesarias.

4. Información perceptible, es decir, que comunique eficazmente la información necesaria para el usuario, según las condiciones ambientales y sensoriales del usuario.

5. Tolerancia al error, es decir, que permita minimizar los riesgos y errores con pleno acceso a los elementos más usados, y eliminando, aislando o cubriendo los elementos peligrosos. Debe proporcionarse advertencias explícitas sobre posibles fallas y peligros.

6. Bajo esfuerzo físico, es decir, que el usuario pueda usarlo eficaz y cómodamente, sin fatigarse.

7. Tamaño y espacio para el acceso y el uso, es decir, que se puedan alcanzar y manipular sus elementos según las condiciones corporales y motrices del usuario. 
La variables y características terapéuticas serán definidas con los profesionales. Esto es lo que se pretende lograr como valor terapéutico, sumado a que sea medible en las escalas clínicas que se definan como requerimientos de diseño. Esta

\section{Tercera fase: implementación del prototipo}

De acuerdo con lo evidenciado en las dos primeras fases, en la tercera se explica cómo funciona de manera general el prototipo construido y se analiza el porcentaje de implementación

\section{Desarrollo del proyecto}

\section{Identificación del diagnóstico.}

Los juegos didácticos pueden estimular diferentes áreas de una persona, más aún en el caso de las personas con discapacidad cognitiva. Mediante una mirada retrospectiva, se evidencia que el prototipo implementado estimula de

\section{Diseño del prototipo.}

De acuerdo con la identificación del diagnóstico (fase 1), se tienen en cuenta las características fundamentales para realizar el diseño de un prototipo cuya interfaz resulte ser intuitiva y amigable, con el objetivo de que el usuario final pueda realizar la actividad. El fin que se quiere alcanzar con el prototipo es que este pueda ser utilizado por niños en edad escolar. intervención está aún por desarrollarse junto a los profesionales de salud. Como se definió al inicio de la metodología, este es un prototipo que se encuentra en etapa de diseño.

alcanzado hasta la actualidad. Se aclara que este proyecto se encuentra en su primera etapa de diseño.

manera superficial los campos correspondientes a la concentración, atención y reacción. En el modelo propuesto se evalúan las competencias de los participantes.

Se consideró que el dispositivo dependiera únicamente de conectar a la energía eléctrica y que no hiciera falta un menú de configuración. A continuación, se presenta, en la figura 1, un diagrama esquemático de bloques, en el que se observan los elementos incorporados en la construcción del prototipo electrónico. 


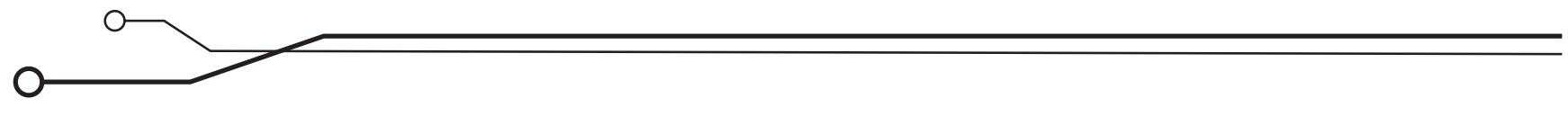

Figura 1. Diagrama de bloques del prototipo electrónico.

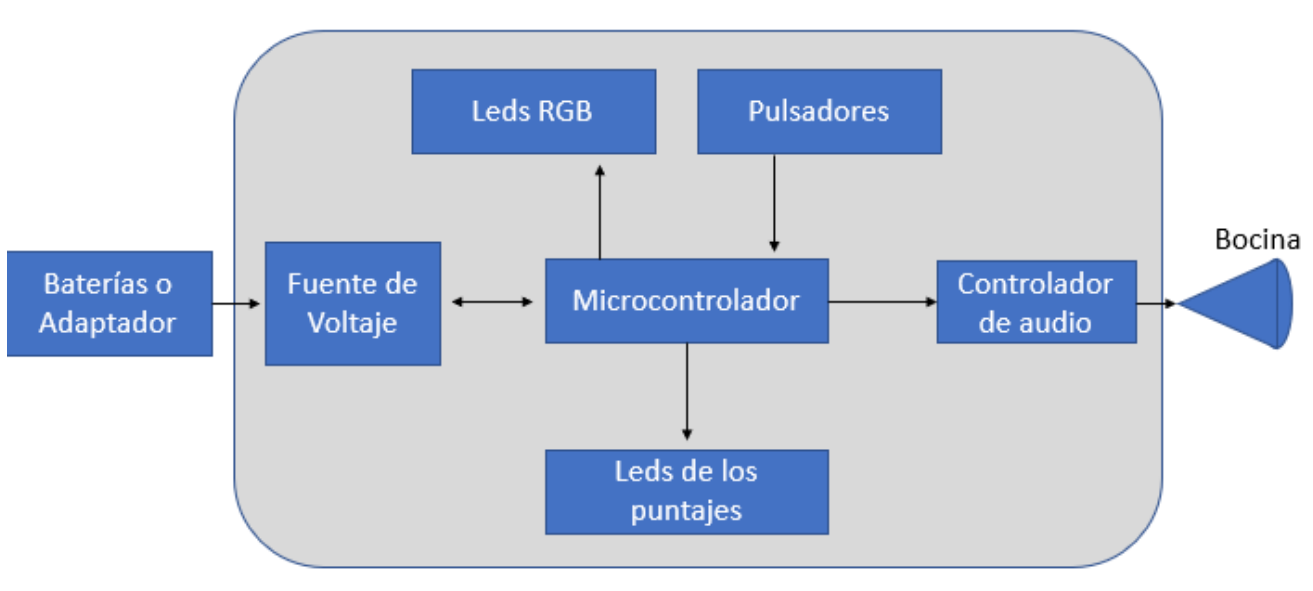

Fuente: elaboración propia.

El prototipo contiene un sistema audiovisual con el fin de que los participantes puedan familiarizarse con el dispositivo como un juego básico, con el que se puedan divertir e interactuar con otros. El enfoque principal del dispositivo pretende evaluar la habilidad de respuesta de dos participantes. Por medio de dos pulsadores conectados a sistema de control, se determina la rapidez con la que cada uno de ellos es capaz de reconocer un indicador.

El controlador, mediante su programación en C, una vez iniciado el juego, emite un tren de pulsos a diferente frecuencia hacia los leds RGB, con el fin de variar su tonalidad. Este analiza constantemente el estado de los pulsadores, que se encuentran en un estado inicial de $+5 \mathrm{~V}$ pero que, cuando son pulsados, cambian a $0 \mathrm{~V}$. El controlador percata esta variación de voltaje y, mediante su lógica de programación, evalúa cuál fue el participante que oprimió en primer lugar el pulsador. Debido a esta acción, el controlador emite un pulso que controla el driver de audio. En un instante de tiempo más, envía otro pulso para encender los leds de los puntos o apagar el led al participante en el caso que de este se equivoque. A continuación, se detalla en la figura 2 el diagrama de flujo que corresponde a la lógica del controlador. 
Figura 2. Diagrama de flujo del controlador.

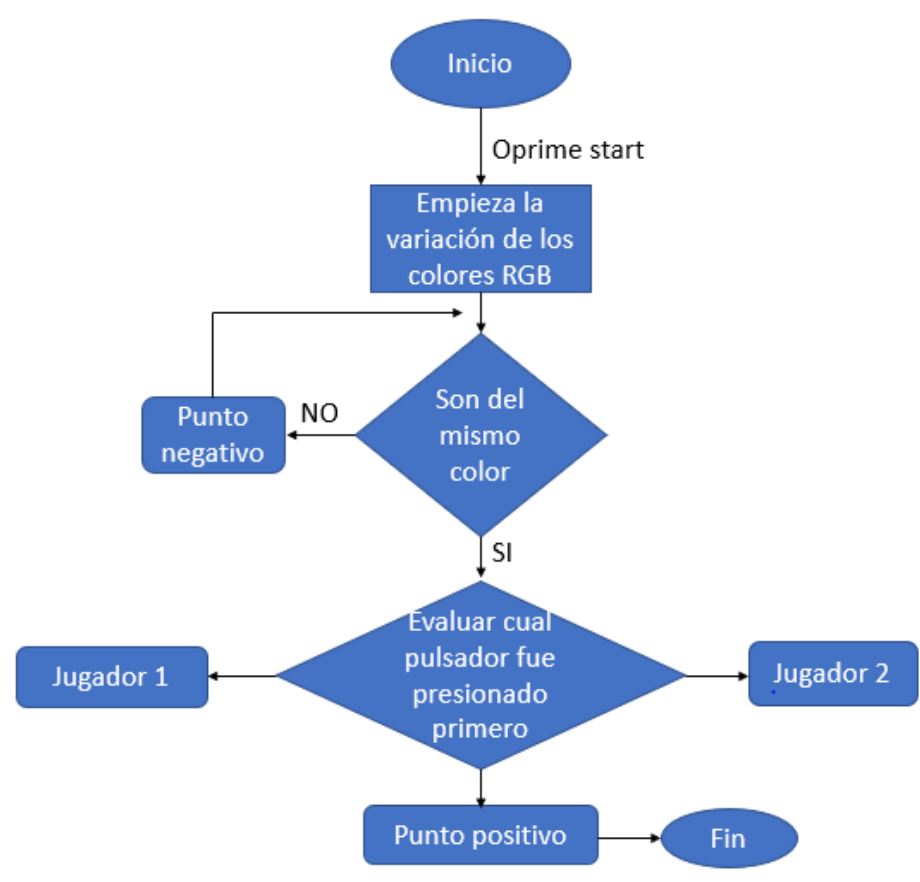

Fuente: elaboración propia.

\section{Implementación del prototipo y dinámica del juego}

En el sistema de control se tienen conectados:

- Dos leds RGB en la parte frontal del prototipo con el fin de que los jugadores puedan siempre observar claramente la tonalidad del color de los leds.

- Dos pulsadores de contacto, ubicados a cada lado de los participantes para poder presionar una vez sea el momento.

- Un pulsador de start.

- Reproductor de Audio.

El juego se inicia con la conexión a la energía eléctrica, ya sea por la conexión de un adaptador de $9 \mathrm{~V}$ - 1A, o también se puede realizar la conexión del dispositivo a una batería de estas mismas características. Una vez se enciende el dispositivo, este cuenta con un reproductor de mp3, que reproduce un audio con la instrucción a los participantes antes de comenzar a jugar. Una vez se ha accionado el pulsador de start, se inicia el juego, partiendo con cierta tonalidad de color en los leds RGB por un tiempo desconocido para los participantes (pero determinado en la programación del controlador). En un instante de tiempo diferente, al primero se le sincronizan los leds RGB (Rojo-Rojo, Verde-Verde, AzulAzul). El jugador que presione el pulsador con mayor velocidad, atención y respuesta, obtendrá un punto positivo. En caso de que el participante presione el pulsador en el momento que las tonalidades de color no estén sincronizadas, este perderá un punto si cuenta con alguno positivo, o en caso de que no tenga puntos se le sumará un punto negativo.

Para poder ganar se deben completar cinco puntos positivos, los cuales se observan en unos leds de lado del participante (Verdes - Puntos 


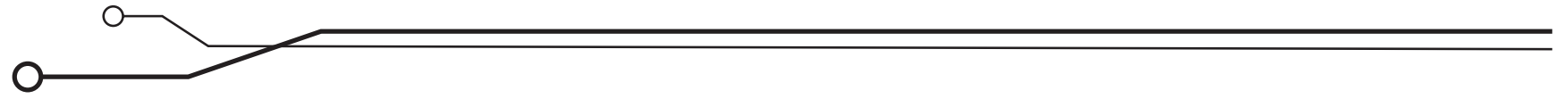

Positivos y Rojos - Puntos Negativos). En caso de que un participante gane un punto positivo, el dispositivo de audio se activa para decirle al participante "bien hecho". En caso de que el punto sea negativo, el sistema de audio se activa para decirle al participante "te equivocaste". Cuando alguno de los dos participantes logra completar cinco puntos correctos, el sistema de audio felicita al ganador y vuelve a reiniciarse para jugar de nuevo.

Figura 3. Prototipo elaborado real.

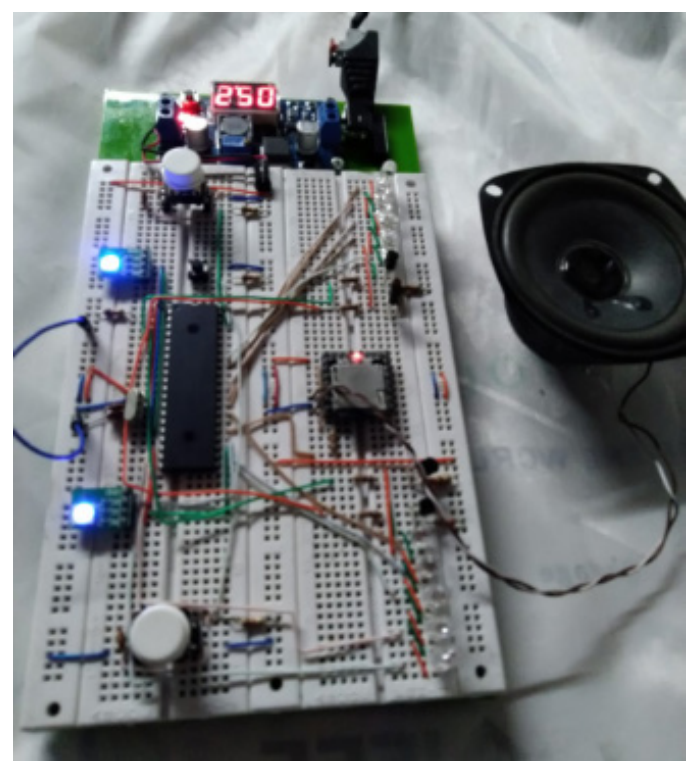

Fuente: elaboración propia.

El prototipo previsto se encuentra en fase experimental y aún no se han realizado pruebas con los usuarios objetivo de este diseño.

\section{Resultados y discusión}

Se plantea que el desarrollo estará sujeto a pruebas de acuerdo a las variables clínicas que se establezcan, y así mejorar el prototipo con interfaces gráficas adaptadas al usuario, un diseño ergonómico, la optimización de funciones (como la de oprimir un pulsador), la visualización en una pantalla táctil, o una mayor interacción con los usuarios, permitiéndoles el acceso a etapas de mayor nivel y que cuenten con un grado de complejidad más alto, así como mayor memoria para guardar resultados y poder evaluarlos de acuerdo a las variables clínicas establecidas.

Con este prototipo se inicia en el grupo IDECUN y en el semillero Electrocun la investigación y el desarrollo de dispositivos lúdicos para personas en condición de discapacidad. Paralelamente a esta experiencia, se construyen pautas de fases preclínicas y clínicas para este tipo de diseños, dentro de lo que sea posible. Se resalta que con la experiencia de la CUN en desarrollos dentro del 
ámbito de la robótica y la electrónica se aporta de manera significativa a esta nueva etapa de exploración de la ingeniería aplicada a la discapacidad, como un soporte y ayuda para brindar a esta población mejor calidad de vida. De igual manera, se vincula a estudiantes y jóvenes investigadores a desarrollar proyectos con enfoque e impacto social como este tipo de dispositivos tecnológicos, con características seguras y adaptables a las condiciones de cualquier usuario.

\section{Referencias}

Arévalo, J., Roa, J. y Galeano, J. (2007). Playtek: juguete electrónico para niños con discapacidad mental. Umbral Científico, 11, 17-28. Recuperado de http://www.redalyc.org/ pdf/304/30401103.pdf

Costa, M., Romero, M., Mallebrera, C., Fabregat, M., Torres, E., Martínez, M., Martínez, Y., Zaragoza, R., Torres, S. y Martínez, P. (2007). Juego, Juguetes y Discapacidad. Alicante: Asociación Investigación Industria del Juguete. Recuperado de http://www.cesya.es/sites/default/ files/documentos/folleto \%20AIJU.pdf

De Miguel, M. (2006). Metodologías de enseñanza y aprendizaje para el desarrollo de competencias. Orientaciones para el profesorado universitario ante el espacio europeo de educación superior. Madrid: Alianza Editorial.

Discpacnet. (s. f.). Tecnología Inclusiva. Fundación ONCE. Disponible en: https:/ / www.discapnet.es/areas-tematicas/tecnologia-inclusiva/guia-de-juguetes-accesibles

Discapacidad Colombia. (2017). Inclusión Educativa. Recuperado de http:/ / www.discapacidadcolombia.com/index.php/inclusion-educativa

Moreno, L., Martínez, P. (2014). Evitando las Barreras de Accesibilidad en la Sociedad de la Información. Universidad Carlos III de Madrid. Recuperado de http://aprende.colombiaaprende.edu.co/sites/default/files/naspublic/cerrandobrechas/importantes/tema-2-diseno-universal.pdf

Organización Mundial de la Salud. (2001). Clasificación Internacional del Funcionamiento, de la Discapacidad y de la Salud: CIF. Versión abreviada. Recuperado de https:/ /apps.who.int/ iris/bitstream/handle/10665/43360/9241545445_spa.pdf

Organización Mundial de la Salud. (2011). Informe mundial sobre la discapacidad. Recuperado de https://www.oas.org/es/sedi/ddse/paginas/documentos/discapacidad/DESTACADOS/ResumenInformeMundial.pdf

Sevilla, G. (2011). La naturaleza relacional entre la discapacidad y el diseño: modelo sistémico de análisis Persona en situación de discapacidad - Entorno construido (tesis de maestría) Universidad Nacional de Colombia, Bogotá. 